\title{
Relationship between Post-Stroke Cognitive Impairment and Functional Activity of Stroke Survivors in Maiduguri, Borno State Nigeria
}

\author{
Abdulsalam Abdullah', Abubakar Umar², M. A. Abdulkadir'1, Muhammad Abdullahi Sa'id Bmr², \\ Jimada Yahaya Danladi ${ }^{3}$
}

${ }^{1}$ Department of Physical and Health Education, Faculty of Education, University of Maiduguri, Maiduguri, Nigeria

${ }^{2}$ Department of Medical Rehabilitation (Physiotherapy), University of Maiduguri, Maiduguri, Nigeria

${ }^{3}$ Department of Social Development, Kaduna Polytechnic, Kaduna South LGA, Nigeria

Email: just2abdul@gmail.com

How to cite this paper: Abdullah, A., Umar, A., Abdulkadir, M. A., Bmr, M. A. S., \& Danladi, J. Y. (2019). Relationship between Post-Stroke Cognitive Impairment and Functional Activity of Stroke Survivors in Maiduguri, Borno State Nigeria. Advances in Physical Education, 9, 8-22. https://doi.org/10.4236/ape.2019.91002

Received: September 16, 2018 Accepted: December 23, 2018 Published: December 26, 2018

Copyright ( 2019 by authors and Scientific Research Publishing Inc. This work is licensed under the Creative Commons Attribution International License (CC BY 4.0).

http://creativecommons.org/licenses/by/4.0/ (c) (i) Open Access

\begin{abstract}
Stroke survivors often experience serious cognitive impairment which may impact on their functional activity and the ability to lead an independent life. However, there is paucity of information on the relationship between post-stroke cognitive impairment (PSCI) and functional activity of Nigerian stroke survivors. This study therefore examined the relationship between post-stroke cognitive impairment (PSCI) and functional activity of stroke survivors attending physiotherapy clinics in Maiduguri, north-eastern Nigeria. A cross sectional study design was utilized and a purposive sampling technique was used to recruit participants for this study. One hundred stroke patients from Physiotherapy clinics in Maiduguri, north-eastern Nigeria participated in this study. Data forms were used to obtain information on socio-demographic and clinical characteristics of the participants while Mini-Mental State Examination (MMSE) and Barthel Index (BI) scale were used to obtain information on cognitive impairment and functional activity of the participants respectively. Descriptive statistics of mean, standard deviation, frequency and percentages were used to summarise the socio-demographic and clinical characteristics of the participants. Spearman's Rank Order Correlation was used to investigate the relationship between PSCI and overall functional activity of the participants on one hand and between PSCI and components of functional activity of the participants. Out of the 100 stroke survivors that participated in the study, 55\% were male while $45 \%$ were female. The mean age and post-stroke duration of the participants were $55.16 \pm 10.01$ years and $9.90 \pm 10.07$ months respectively. The results showed that a positive and significant relationship existed between PSCI and
\end{abstract}


overall functional activity of the participants $(\mathrm{r}=0.55, p=0.00)$. Similarly, there was a significant and positive correlation between PSCI and the components of functional activity except with the bowel control component of functional activity. The correlations were varied with the highest correlation observed between PSCI and mobility component $(\mathrm{r}=0.58, p=0.001)$ while the lowest correlation was between PSCI and the bowel control component $(\mathrm{r}=$ $0.17, p=0.10$ ). the correlation coefficient for the other components namely the feeding, bathing, grooming, dressing, bladder control, toilet use, transfer and stair climbing were $0.40,0.45,0.40,0.41,0.39,0.44,0.50$ and 0.53 respectively. The outcome of this study showed that the relationship between PSCI and functional activity of the stroke survivors was significant and positive. Furthermore, correlation was highest between PSCI and mobility component of functional activity. It is concluded that reducing PSCI could positively impact on the functional activity of stroke survivors, and enhancing functional activity could also reduce Post-Stroke Cognitive Impairment (PSCI) and therefore Physiotherapists should endeavor to assess cognitive impairment among stroke patients and incorporate treatment strategies that will enhance functional outcome.

\section{Keywords}

Post-Stroke Cognitive Impairment, Functional Activity, Stroke Survivors

\section{Introduction}

Stroke, also known as cerebrovascular accident (CVA) is a rapidly developing loss of brain function due to disturbance in the blood supply to the brain. The disturbance in blood supply can be due to ischemia caused by blockage from thrombosis, arterial embolism, or a hemorrhage (Sims \& Muyderman, 2009). Loss of brain function could result to inability to move one or more limbs of one side of the body, inability to understand or formulate speech, or an inability to see on one side of the visual field depending on the area of the brain affected (Donnan et al., 2008).

Stroke is the leading cause of disability in both the developed and the developing world (Feign et al., 2009) and results in significant impairment and activity limitation in many functional domains (Hommel et al., 2009). Reports showed that within 12 months after stroke, approximately $37 \%$ of patients will die and $10 \%$ will experience a recurrent stroke. Stroke can also result to a wide range of deficits affecting cognitive functions both during the acute and long term (Nys et al., 2005). Age, functional status, co-morbidities, and cognitive functions are known to be the predictors of functional outcome in stroke (Denti et al., 2008).

Post stroke cognitive impairment (PSCI) is a mental deficit that usually develops during three months after stroke with a minimal duration of six months which is not associated with any other condition or disease like depression, metabolic and endocrine disorders (Viscogliosi et al., 2011). Cognitive impairment 
includes impairments in domains such as memory, attention, executive function, language, praxis and perfection (Robert \& Norhayati, 2014). PSCI can be attributable to damage to the cortical regions of the brain which leads to disruption of neuronal function at remote cerebral regions that belong to certain functional networks such as frontal (pre frontal gyrus) and temporal lobes (Rosalia et al., 2014; Crofts et al., 2011). Nearly one in four stroke patients have cognitive impairment severe enough to be diagnosed as dementia, while one in three has milder levels of cognitive impairment. When cognitive deficits are not identified and compensated for, they could lead to restrictions of daily life activities (Viscogliosi et al., 2011).

Functional activity can be described as an activity that is essential to support the physical, social and psychological well-being of a person and allows that person to function independently in the society (Farlex Medical Dictionary for the Health Profession and Nursing, 2012). Approximately two thirds of stroke patients have residual neurological deficits that impair function and 50\% are left with disabilities thereby making them dependent on others for activities of daily living (Sachdev et al., 2009). Maya et al. (2012) found that substantial percentage of stroke patients demonstrated restrictions in some functional areas; this include: basic activities of daily living (BADL)-33\%, household task (51\%), travel (50\%) and meaningful activities (in the social, recreational and occupational domains) (53\%). The finding concludes that the population is at risk for a diminished functional activity level and social isolation which can negatively impact on their health.

The negative impact of post-stroke cognitive impairment (PSCI) on functional outcome of stroke survivors has been reported in a number of studies. The findings for instance from a study in Romania on the impact of the cognitive impairment after stroke on ADL revealed a strong impact of cognitive impairment on most domains of functional activity after stroke (Oros et al., 2016). Nurdan et al. (2010) in Turkey studied the impact of cognitive impairment on functional outcome in stroke survivors and reported that cognitive dysfunction interfered with $\mathrm{ADL}$ and global recovery in stroke survivors. Another study in Hong-Kong on cognitive impairment and functional outcome after stroke revealed that cognitive dysfunction contributed most to a poor functional outcome (Mok et al., 2004). Rosalia et al. (2014) reported that ability to regulate activities of daily living is directly proportional to normal brain function after stroke. In Nigeria, there is no readily available report on how PSCI relates to the overall domains of functional activity after stroke. A few studies are however known to have examined the relationship between PSCI and functional outcome in stroke survivors (Oros et al., 2016). In Nigerian stroke survivors, there appear to be no known study particularly in the North eastern Nigeria. This study therefore sought to investigate the relationship between PSCI and functional activity level of stroke survivors attending Physiotherapy Clinics in Maiduguri Borno state, north-eastern Nigeria. 


\section{Significance of the Study}

This study may provide information about the relationship between PSCI and functional activity of stroke survivors attending physiotherapy clinics in Maiduguri. This information may assist in the formulation of intervention strategies which will improve functional activity level of stroke survivors. It may also stress the need to include assessment of cognitive impairment and to incorporate appropriate intervention strategies to improve functional outcome of stroke survivors.

\section{Methods}

Participants; The participants for this study were stroke survivors, attending Physiotherapy Clinics of the University of Maiduguri Teaching Hospital and State Specialist Hospital, Maiduguri, Borno State, North eastern Nigeria. Those who are diagnosed with acute disorders like cancer and tumours, willing to participate in the study or suffering from intellectual disability were excluded from the study. Furthermore, those with impaired level of consciousness, aphasia or insufficient communication skill, visual loss and history of depression or drug abuse were excluded from the study

\section{Instruments}

\subsection{Participant's Data Form}

The socio-demographic and clinical data of all stroke patients were documented using the data forms. The forms were developed by the researcher and were used to obtain information on the participant's age, gender, marital status, level of education, side of stroke, post-stroke duration, employment status and nature of stroke whether first or recurrent.

\subsection{The Mini-Mental State Examination (MMSE)}

The MMSE was used to assess the cognitive impairment among stroke survivors. It was developed by Folstein et al. (1975) and it is widely used with small modification of the test and its items. It is an 11-item scale that tests five areas of cognitive function. They are: orientation, registration, attention and calculation, recall and language. The test yields a total score of 30 with a score of 23 or less generally accepted as an indication of cognitive impairment. The levels of impairment have also been classified as none (24 - 30) mild (18 - 24) and severe (0 17) (Tombaugh \& McIntyre, 1992). The scales internal consistency was adequate-alpha $=0.78$ test-retest reliability ranges from poor to excellent $(r>0.75)$ (Tombaugh \& McIntyre, 1992). Molloy and Standish (1997) examined the intra-rater reliability ICC of 0.69 and Dick et al. (1984) examined the inter-rater reliability and reported a kappa of 0.63 , demonstrating the adequate reliability of the MMSE. It has been reported that the MMSE demonstrates marked ceiling effects in younger intact individuals and marked floor effects in individuals with 
moderate to severe impairments. Low levels of sensitivity have been reported particularly among individuals with mild cognitive impairment (De Koning et al., 1998) and may be due to the emphasis placed on language items and lack of items assessing visual spatial ability. The MMSE takes only 5 - 10 minutes to administer and is therefore practical to use repeatedly and respectively. Since its creation in 1975, the MMSE has been validated and extensively used in both clinical practice and research.

\subsection{The Barthel Index (BI)}

It was used to assess the functional activity of the participants in the study. It is an ordinal scale used to measure performance in activities of daily living (ADL). Each performance is rated on this scale with a given number of points assigned to each level. A score between 0 - 20 indicates total dependence, 21 - 60 indicates severe dependence, 61 - 90 indicate moderate dependence and 100 indicate independence. The scale has 10 items measuring patient's level of function; they include bowel continence, bladder continence, grooming, toilet use, feeding, transfer, mobility, dressing, stairs climbing and bathing

Most recently, the Barthel index was found to be reliable in assessing post stroke patients (Crombach's range $0.4-0.85$ ). Barthel index consistency has been described as good (alpha $0.8-0.89$ ) to excellent (0.93). Validity was found to be 0.73 and 0.77 . Responsiveness across a certain range of post stroke disability is reasonable. The Barthel index is more sensitive to change than other common stroke scales (Hseuh et al., 2002).

\section{Study Sites}

This research was conducted at the Physiotherapy Clinics of University of Maiduguri Teaching Hospital (UMTH), Maiduguri and Borno State Specialist Hospital, Maiduguri, Borno State, North-Eastern Nigeria.

Sampling Technique: A purposive sampling technique was utilized in recruiting participants for the study. Male and female hemiplegic stroke survivors who meet the inclusion criteria were recruited from the Physiotherapy Clinics in Maiduguri, Borno state.

\section{Procedure}

The approval of Research and Ethical Committee of the University of Maiduguri Teaching Hospital (UMTH) and official permission of the Heads of Department of Physiotherapy of the University of Maiduguri Teaching Hospital (UMTH) and State Specialist Hospital, Maiduguri were sought and obtained before the commencement of this study. Participants' consent was obtained before their participation in the study. The questionnaires were administered to the participants directly by the researcher and filled out at the study site and retrieved on the same day. 


\section{Data Analyses}

1) Descriptive statistics of mean, standard deviation and percentages were used to summarize the socio-demographic data of participants.

2) Spearman correlation co-efficient was used to determine the relationship between post-stroke cognitive impairment and:

a) Domains of functional activity of stroke survivors attending Physiotherapy Clinics in Maiduguri.

b) "Bowel" control domain of functional activity of stroke survivors attending Physiotherapy Clinics in Maiduguri.

c) "Bladder control" domain of functional activity of stroke survivors attending Physiotherapy Clinics in Maiduguri.

d) "Grooming" domain of functional activity of stroke survivors attending Physiotherapy Clinics in Maiduguri.

e) "Toilet use" domain of functional activity of stroke survivors attending Physiotherapy Clinics in Maiduguri.

f) "Feeding" domain of functional activity of stroke survivors attending Physiotherapy Clinics in Maiduguri.

g) "Transfer" domain of functional activity of stroke survivors attending Physiotherapy Clinics in Maiduguri.

h) "Mobility" domain of functional activity of stroke survivors attending Physiotherapy Clinics in Maiduguri.

i) "Dressing" domain of functional activity of stroke survivors attending Physiotherapy Clinics in Maiduguri.

j) "Stairs" domain of functional activity of stroke survivors attending Physiotherapy Clinics in Maiduguri.

k) "Bathing" domain of functional activity of stroke survivors attending Physiotherapy Clinics in Maiduguri.

Level of statistical significance was set at 0.05 alpha of significance.

\section{Results}

A total of 106 stroke survivors were approached for this study from the Physiotherapy Departments of the University of Maiduguri Teaching Hospital and the State Specialist Hospital, Maiduguri, Borno State, out of which six (6) of the stroke patients were excluded thereby resulting to a response rate of $94.3 \%$. Three (3) were excluded because they refuse to participate, two (2) were aphasic and another one (1) excluded due to visual problem all of which have an influence on response the participants will give for the questionnaires.

\subsection{Socio-Demographic and Clinical Characteristics of Participants}

100 stroke survivors participated in this study, 55 (55\%) of whom were males and $45(45 \%)$ were females. The mean age of the participants was $55.16 \pm 10.01$ (range $=23-83$ ) years, and the post-stroke duration of the participants was $9.90 \pm$ 
10.07 (range $=0.25$ - 48) months. Majority of the participants were suffering from right sided hemiplegia (52\%), as were those with the first incidence (98\%), base on marital status the percentage of those who married was higher (62\%), those employed were higher (51\%) than those unemployed, and base on educational status those with Qur'anic education only were higher (47\%) (Table 1).

\subsection{Relationship between Post-Stroke Cognitive Impairment and Functional Activity of the Participants}

Table 2 shows the results of the correlation analysis between scores of the participants on the Mini-Mental State Examination (MMSE) and the Barthel Index

(BI) scale. A positive and significant relationship was found between post-stroke cognitive impairment and overall functional activity of stroke survivors $(\mathrm{r}=$ $0.55, p=0.00)$.

\subsection{Relationship between Post-Stroke Cognitive Impairment and Components of Functional Activity of the Participants}

Positive and significant correlations were found between scores on the

Table 1. Socio-demographic characteristics of the stroke survivors.

\begin{tabular}{|c|c|c|c|}
\hline \multirow[t]{2}{*}{ Variable } & \multirow[t]{2}{*}{ Mean \pm SD (Range) } & \multicolumn{2}{|c|}{ Frequency } \\
\hline & & $\mathrm{n}$ & $\%$ \\
\hline Age (years) & $55.16 \pm 10.01(23-83)$ & & \\
\hline PSD (month) & $9.90 \pm 10.07(0.25-48)$ & & \\
\hline \multicolumn{4}{|l|}{ Gender } \\
\hline Male & & 55 & 55 \\
\hline Female & & 45 & 45 \\
\hline \multicolumn{4}{|l|}{ Marital Status } \\
\hline Single & & 3 & 3 \\
\hline Married & & 62 & 62 \\
\hline Divorced & & 16 & 16 \\
\hline Widowed & & 19 & 19 \\
\hline \multicolumn{4}{|l|}{ Educational Status } \\
\hline Secondary & & 13 & 13 \\
\hline Tertiary & & 27 & 27 \\
\hline Qur'anic & & 47 & 47 \\
\hline None & & 13 & 13 \\
\hline \multicolumn{4}{|c|}{ Employment Status } \\
\hline Employed & & 51 & 51 \\
\hline Unemployed & & 42 & 42 \\
\hline Retired & & 7 & 7 \\
\hline \multicolumn{4}{|c|}{ Body side affected } \\
\hline Right & & 52 & 52 \\
\hline Left & & 48 & 48 \\
\hline \multicolumn{4}{|l|}{ Nature of Stroke } \\
\hline First & & 98 & 98 \\
\hline Recurrent & & 2 & 2 \\
\hline
\end{tabular}

Key: $\mathrm{PSD}=$ post-stroke duration; $\mathrm{n}=$ number of participants; $\%=$ percentage 
Table 2. Spearman rank order correlation between Mini-Mental State Examination (MMSE) scores and overall and components scores of the Barthel Index (BI) scale.

\begin{tabular}{ccc}
\hline Variable & MMSE (Total) Rho & $p$-value \\
\hline BI (Total score) & 0.55 & 0.001 \\
Feeding & 0.44 & 0.001 \\
Bathing & 0.45 & 0.001 \\
Grooming & 0.40 & 0.001 \\
Dressing & 0.41 & 0.001 \\
Bowel control & 0.17 & 0.10 \\
Bladder control & 0.39 & 0.001 \\
Toilet use & 0.44 & 0.001 \\
Transfer & 0.50 & 0.001 \\
Mobility & 0.58 & 0.001 \\
Stair climbing & 0.53 & 0.001 \\
\hline
\end{tabular}

KEY: MMSE = Mini-Mental State Examination; BI = Barthel Index; rho = Correlation Coefficient.

Mini-Mental State Examination (MMSE) and scores on the components of functional activity such as feeding, bathing, grooming, dressing, bladder control, toilet use, transfer, mobility and stair climbing, and were found to be not significant with the bowel control component of the Barthel Index (BI) scale (Table 2).

\section{Discussion}

\subsection{Socio-Demographic and Clinical Characteristics of the Participants}

One hundred volunteer ambulatory hemiplegic stroke survivors participated in this study from the Physiotherapy Departments of the University of in Maiduguri Teaching Hospital (UMTH) and the State Specialist Hospital, North-eastern Nigeria. The gender of the participants in this study was virtually evenly distributed among male and female participants constituting 55\% and $45 \%$ respectively, which is in line with findings of several studies in both the developed and the developing countries in which even distribution of participants gender was reported among stroke survivors (Oros et al., 2016). The mean age of the participants was $55.16 \pm 10.01$ and the range was $23-83$ years, which is similar to the mean age of $55.97 \pm 9.56$ years reported in a study by Vincent-Onabajo \& Moses (2016). Majority of the participants were married (62\%) as were those employed (51\%) which is in line with findings by Vincent-Onabajo and Adamu (2014). The majority of the participants attended Qur'anic education, this was inconsistent to findings by Oros et al. (2016) who reported that majority of the participants had tertiary education. This inconsistency could be due to the fact that the predominant type of education in the North-eastern Nigeria especially Maiduguri is the Qur'anic education as reported by Naijainformans notes (2012) or could be due to the fact that there are no many tertiary institutions in the region. 


\subsection{Relationship between Post-Stroke Cognitive Impairment and Overall Functional Activity of the Participants}

A significant and positive correlation was found between Mini-Mental State Examination (MMSE) scores and overall Barthel Index (BI) score. The positive relationship between post-stroke cognitive impairment (PSCI) and overall functional activity indicates that a high level of cognitive impairment is associated with diminished functional activity and vice-versa. This finding is consistent with that of a previous study in which post-stroke cognitive impairment correlated significantly and positively with overall functional activity of stroke survivors (Oros et al., 2016). Also, another study reported the existence of significant correlation between Mini-Mental State Examination (MMSE) and Blessed Functional Activity Scale (BFAS), Barthel Index (BI) and the Stroke Severity Scale (SSS) as measures of functional activity (Tatemichi et al., 1994).

In a similar study by Nurdan et al. (2010), the impact of cognitive impairment on functional outcome after stroke was investigated and a correlation between Mini-Mental State Examination (MMSE) and the Barthel Index measure of functional activity ranging from $r=0.21$ to 0.73 was reported. However, in this present study, correlation between PSCI and functional activity was different $(\mathrm{r}=0.17$ to 0.58$)$ from reports of Nurdan et al. (2010). This difference may be because PSCI has less impact on the functional activity of stroke survivors that participated in this study.

\subsection{Relationship between Post-Stroke Cognitive Impairment and Feeding Component of Functional Activity among the Participants}

The feeding component of functional activity correlated significantly and positively with cognitive impairment among the participants, the relationship could have occurred due to the deficits in cognitive function domain such as attention especially focus attention, or visuo-perception problems as reported by Nys et al. (2005). It may also be due to hemineglect or behavioural problems experienced by stroke survivors which are important predictors of functional outcome including feeding post-stroke (Robert and Norhayati, 2014).

\subsection{Relationship between Post-Stroke Cognitive Impairment and Bathing Component of Functional Activity among the Participants}

The finding from this study on the relationship between PSCI and bathing component of functional activity revealed a significant and positive correlation. This is in line with findings from Stephens et al. (2005) which found that PSCI impacts negatively on one's ability to carry out some activities of daily living such as bathing, and may be attributable to cognitive deficits which result to physical incapacity, motor, behavioural and executive function deficits such as initiation, problem solving and planning as reported by Port et al. (2008). It may 
also be due to hemineglect and reduced muscle strength associated with stroke (Rhoda, 2012).

\subsection{Relationship between Post-Stroke Cognitive Impairment and Grooming Component of Functional Activity among the Participants}

The finding of this study found significant and positive relationship between PSCI and grooming component of functional activity among stroke survivors. Relationship was observed with a correlation coefficient of $(r=0.45)$, which may be attributable to inattention, apraxia, agnosia, executive function problem as reported by Stephens et al. (2005). Other factors such as hemineglect reduced motor strength and poor motor skills may lead to limitation in self-care activities such as grooming in the presence of cognitive impairments as reported in a study by Rhoda (2012).

\subsection{Relationship between Post-Stroke Cognitive Impairment and Dressing Component of Functional Activity among the Participants}

A significant and positive relationship was found between PSCI and the dressing component of functional activity of the participants with a correlation coefficient of $(\mathrm{r}=0.41)$, which is similar with findings from other studies (Oros et al., 2016; Nurdan et al., 2010). The significant relationship between PSCI and dressing among the participants could be due to the attention loss, ataxia and executive function problems associated with post-stroke cognitive impairment (Nurdan et al., 2010). The relationship could also be as a result of hemineglect, poor social cognition and deficits in muscle strength as reported by Bohannon \& Walsh (1991).

\subsection{Relationship between Post-Stroke Cognitive Impairment and Bowel Component of Functional Activity among the Participants}

There was no significant relationship between post-stroke cognitive impairment and the bowel control component of functional activity among stroke survivors, this finding is contrary to previous studies (Rhoda, 2012), which reported a significant relationship between the variables. This inconsistency could be as a result of high impact of cognitive impairment on the stroke survivors that participated in the previous studies or differences in post-stroke duration (PSD), as the PSD for the participants of this study was $9.90 \pm 10.01$, and bowel incontinence usually ensues in the acute phase of stroke usually $<2$ weeks in about $41 \%-52 \%$ of stroke patients as reported by Krogh et al. (2009). The relationship between PSCI and bowel incontinence may be due to reduced intestinal peristalsis which has been identified as a risk factor for intestinal constipation experienced by stroke patients as reported by Tania et al. (2014) which may lead to deconditioning or inappropriate abdominal press strength for defecation. 


\subsection{Relationship between Post-Stroke Cognitive Impairment and Bladder Component of Functional Activity among the Participants}

There was a significant and positive relationship between post-stroke cognitive impairment and the bladder control component of functional activity of stroke survivors with a correlation coefficient of $(r=0.39)$. Poor bladder control is common after stroke and is associated with cognitive deficits such as attention loss, perception and behavioural problems which in turn affects overall functional outcome after stroke (Oros et al., 2016). Finding from this study is similar to finding from a study by (Nurdan et al., 2010) which showed that poor bladder control is associated with severe cognitive deficits among stroke survivors, this could also be as a result of speech alteration besides cognitive impairment object agnosia, communication and self-awareness problem, abstract thinking, social cognition problems and reasoning deficits which can compromise evacuation at socially acceptable time and place as reported by Rhoda (2012).

\subsection{Relationship between Post-Stroke Cognitive Impairment and Toilet Use Component of Functional Activity among the Participants}

Post-stroke cognitive impairment (PSCI) and toilet use component of functional activity correlated significantly as found by this study, which is in line with findings from studies by Rhoda et al. (2012). This correlation could be due to mild attention loss, disorientation, executive function deficits, visuo-perceptual problems, visuo-spatial problems and lack of self awareness (Stephens et al., 2005). It may also be limited by the reduced motor skills, muscle strength and hemineglect experienced by stroke survivors (Bohannon \& Walsh, 2007).

\subsection{Relationship between Post-Stroke Cognitive Impairment and Transfer Component of Functional Activity among the Participants}

There was a significant and positive relationship between post-stroke cognitive impairment and the transfer component of functional activity of stroke survivors with a moderate level of correlation (0.50). This finding is consistent with previous findings (Oros et al., 2016; Nurdan et al., 2010). This may be attributable to the attention deficits and executive dysfunction (Nys et al., 2005; Stephens et al., 2005). It may also be limited by the reduced motor skills, muscle strength and hemineglect experienced by stroke survivors (Bohannon \& Walsh, 2007).

\subsection{Relationship between Post-Stroke Cognitive Impairment and Mobility Component of Functional Activity among the Participants}

There was a significant and positive relationship between post-stroke cognitive impairment and the mobility component of functional activity of stroke survivors from the findings of this study, which is consistent with previous findings by Oros et al. (2016) and Nurdan et al. (2010). The correlation may be due to 
attention loss, executive function, reasoning, visuo-spatial and visuo-perceptual problems (Stephens et al., 2005), physical incapacity as reported by Rhoda (2012), poor walking speed, balance, endurance and/or use of walking aid (Port et al., 2008) or reduced motor skills, muscle strength and hemineglect manifesting after stroke as reported be (Bohannon \& Walsh (2007).

\subsection{Relationship between Post-Stroke Cognitive Impairment and Stair Climbing Component of Functional Activity among the Participants}

There was a significant and positive relationship between post-stroke cognitive impairment and the stair climbing component of functional activity of stroke survivors which may be attributable to inattention, apraxia, visuo-spatial problems, abstract thinking and memory problems as reported by Nys et al. (2005) and Stephens et al. (2005). It could also be as a result of physical incapacity, poor motor skills, strength and hemineglect associated with stroke (Bohannon \& Walsh, 2007).

\section{Conclusion}

1) A significant and positive relationship existed between post-stroke cognitive impairment (PSCI) and overall functional activity of the stroke survivors.

2) Relationship between post-stroke cognitive impairment and each of feeding, bathing, grooming, dressing, bladder control, toilet use, transfer, mobility and stair climbing components of functional activity of stroke patients were significant and positive, while the bowel control component was not significant.

3) Relationship between PSCI and the components of functional activity varied with the highest correlation observed between PSCI and the mobility component while the lowest correlation existed between PSCI and the bowel control component of functional activity.

\section{Recommendations}

1) Clinicians should create a means of emphasis on interaction with stroke patients to assess cognitive function and counsel them on programs to eliminate post-stroke cognitive impairment as this may impact positively on the functional outcome of the patients.

2) Intervention should be designed for patients with cognitive impairment with consideration for the mobility and self-care components with functional activity to achieve optimal function.

3) Future research on the relationship between post-stroke cognitive impairment and functional activity among stroke survivors should be conducted with a larger sample size in different parts of Nigeria to provide more comparable and generalizable data.

\section{Conflicts of Interest}

The authors declare no conflicts of interest regarding the publication of this paper. 


\section{References}

Bohannon, R. W., \& Walsh, S. (1991). Association of Paretic Lower Extremity Muscle Strength and Standing Balance with Stair-Climbing Ability in Patients with Stroke. Journal of Stroke and Cerebrovascular Diseases, 1, 129-133. https://doi.org/10.1016/S1052-3057(10)80004-7

Crofts, J.J., Higham, D.J., Bosnell, R., Jbabdi, S., Mathews, P.M., Behrens, T.E. and Johansen-Berg, H. (2011). Network Analysis Detects Changes in the Contralesional Hemisphere Following Stroke. Neuro-Imaging, 54, 161-169. https://doi.org/10.1016/j.neuroimage.2010.08.032

Denti, L., Agosti, M., \& Franceschini, M. (2008). Outcome Predictors of Rehabilitation for First Stroke in the Elderly. European Journal of Physical and Rehabilitation Medicine, 44, 3-11.

Dick, J. P., Guiloff, R. J., Stewart, A., Blackstock, J., Bielawska, C., Paul, E. A., \& Marsden, C. D. (1984). Mini-Mental State Examination in Neurological Patients. Journal of Neurology, Neurosurgery and Psychiatry, 47, 496-499. https://doi.org/10.1136/jnnp.47.5.496

De Koning, I., Van Kooten, F., Dippel, D. W., Van Harskamp, F., Grobbee, D. E., Kluft, C., \& Koudstaal, P.J. (1998). The CAMCOG: A Useful Screening Instrument for Dementia in Stroke Patients. Stroke, 29, 2080-2086. https://doi.org/10.1161/01.STR.29.10.2080

Donnan, G. A., Fisher, M., MacLeod, M., \& Davis, S. M. (2008). Stroke. Lancet Neurology, 371, 1612-1623. https://doi.org/10.1016/S0140-6736(08)60694-7

Feign, V. L., Lawes, C. M., Bennett, D. A., Barker-Collo, S. L., \& Parag, V. (2009). Worldwide Stroke Incidence and Early Case Fatality Reported in 56 Population-Based Studies: A Systematic Review. Lancet Neurology, 8, 355-369. https://doi.org/10.1016/S1474-4422(09)70025-0

Folstein, M. F., Folstein, S. E., \& McHugh, P. R. (1975). Mini-Mental State: A Practical Method for Grading the Cognitive State of Patients for the Clinician. Journal of Psychiatry Research, 12, 189-198. https://doi.org/10.1016/0022-3956(75)90026-6

Hommel, M., Miguel, S. T., Naegele, B., Gonnet, N., \& Jaillard, A. (2009). Cognitive Determinants of Social Functioning after a First Ever Mild to Moderate Stroke at Vocational Age. Journal of Neurology, Neurosurgery, and Psychiatry, 80, 876-880. https://doi.org/10.1136/jnnp.2008.169672

Hseuh, I. P., Mao H. F., Huang, H. L., \& Hsieh, C. L. (2002). Comparison of Responsiveness and Predictive Validity of Two Balance Measures in Stroke Patients Receiving Rehabilitation in China. Journal Formos Medical Association, 5, 261-268.

Krogh, K., \& Christensen, P. (2009). Neurogenic Colorectal and Pelvic Floor Dysfunction. Best Practice Research in Clinical Gastroenterology, 23, 531-543. https://doi.org/10.1016/j.bpg.2009.04.012

Maya, D., Boyko, S., Margarita, A., \& Dora, P. (2012). Post-Stroke Cognitive Impairment-Phenomenology and Prognostic Factors. Journal of IMAB, 18, 290-297.

Molloy, D. W., \& Standish, T. I. M. (1997). A Guide to the Standardized Mini-Mental State Examination. International Psycho Geriatrics, 9, 87-94. https://doi.org/10.1017/S1041610297004754

Mok, V. T., Wong, A., Lam, W. W. M., Fan, Y. H., Tang, W. K., Kwok, T., Hui, A. C. F., \& Wong, K. S. (2014). Cognitive Impairment and Functional Outcome after Stroke Associated with Small Vessel Diseases. British Medical Journal, 75, 210-217.

Nurdan, P., Derya, B., Dement, T., Betul, K., \& Caglayan, D. (2010). Impact of Cognitive 
Impairment on Functional Outcome in Stroke. Stroke Research and Treatment, 2010, Article ID: 652612.

Naijainformans Notes (2012). History of Almajiri Educational System. http://www.Naijainformanwordpress.com

Nys, G. M., Van Zandvoort, M. J., \& De Kort, P. L. (2005). The Prognostic Value of Domain Specific Cognitive Abilities in Acute First Ever Stroke. Neurology, 64, 821-827. https://doi.org/10.1212/01.WNL.0000152984.28420.5A

Oros, R. I., Popescu, C. A., Iova, C. A., Mihancea, P., \& Iova, S. O. (2016). The Impact of Cognitive Impairment after Stroke on Activities of Daily Living. Human and Veterinary Medicine International Journal of the Bioflux Society, 8, 41-44.

Port, I. G., Kwakkel, G., \& Lindeman, E. (2008). Community Ambulation in Patients with Chronic Stroke: How Is It Related to Gait Speed? Journal of Rehabilitation Medicine, 40, 23-27. https://doi.org/10.2340/16501977-0114

Rhoda, A. (2012). Limitations in Activity and Participation Experienced by Stroke Patients: A Qualitative Inquiry. South African Journal of Physiotherapy, 68, 20-24. https://doi.org/10.4102/sajp.v68i3.20

Robert, T., \& Norhayati, H. (2014). Rehabilitation of Cognitive Impairment Post-Stroke (pp. 1-41). Stroke Rehabilitation Clinician Handbook.

Rosalia, D., Grana, M., Fernandez-Andujar, M., Lopez-Cancio, E., Caceres, C., Bargallo, N., Barrios, M., Clemente, I., Metserrat, P. T., Sas, M. A., Davalos, A., Auer, T., \& Mataro, M. (2014). Structural Integrity of the Contralesional Hemisphere Predicts Cognitive Impairment in Ischemic Stroke at 3 Months. PLoS ONE, 9, e86119.

Sims, N. R., \& Muyderman, H. (2009). Mitochondria, Oxidative Metabolism and Cell Death in Stroke. Biochemica et Biophysica Acta, 1802, 80-91. https://doi.org/10.1016/j.bbadis.2009.09.003

Sachdev, P., Chen, X., Brodat, H., Thompson, C., Altendorf, A., \& Wen, W. (2009). The Determinants of Longitudinal Cause of Post-Stroke Mild Cognitive Impairment. Journal of the International Neuropsychological Society, 15, 915-923. https://doi.org/10.1017/S1355617709990579

Stephens, S., Kenny, R. A., Rowan, E., Kalaria, R. N., Bradbury, M., \& Pearce, R. (2005). Association between Mild Vascular Cognitive Impairment and Impaired Activities of Daily Living in Older Stroke Survivors without Dementia. Journal of the American Geriatrics Society, 53, 103-107. https://doi.org/10.1111/j.1532-5415.2005.53019.x

Tania, M. N., de Engler, M., Cinthia, C. D., Thias, G. A., Luciano, F., Paulo, A. M., \& Marcelo, P. C. P. (2014). Stroke: Bowel Dysfunction in Patients Admitted for Rehabilitation. The Open Nursing Journal, 8, 43-47.

https://doi.org/10.2174/1874434601408010043

Tatemichi, T. K., Desmond, D. W., Stern, Y., Paik, M. O., Sano, M., \& Bagiella, E. (1994). Cognitive Impairment after Stroke, Frequency, Pattern, and Relationship to Functional Abilities. Journal of Neurology, Neurosurgery and Psychiatry, 57, 202-207. https://doi.org/10.1136/jnnp.57.2.202

Tombaugh, T. N., \& McIntyre, N. J. (1992). The Mini-Mental State Examination: A Comprehensive Review. Journal of American Geriatric Society, 40, 922-935. https://doi.org/10.1111/j.1532-5415.1992.tb01992.x

Viscogliosi, C., Belleville, S., Desrosiers, J., Caron, C. D., \& Ska, B. (2011). BRAD Group, Participation after Stroke, Changes over Time as a Function of Cognitive Deficits. Archives of Gerontology Geriatrics, 52, 36-43.

https://doi.org/10.1016/j.archger.2010.04.020 
Vincent-Onabajo, G., \& Adamu, A. (2014). Impact of Post-Stroke Fatigue on Health Related Quality of Life of Nigerian Stroke Survivors. Journal of Stroke, 16, 195-201. https://doi.org/10.5853/jos.2014.16.3.195

Vincent-Onabajo, G., \& Moses, T. (2016). Knowledge of Stroke Risk Factors among Stroke Survivors in Nigeria. Stroke Research and Treatment, 2016, Article ID: 1902151. 